\title{
THE FINAL FATE OF STARS THAT IGNITE NEON AND OXYGEN OFF-CENTER: ELECTRON CAPTURE OR IRON CORE-COLLAPSE SUPERNOVA?
}

\author{
SAmuel Jones ${ }^{1,2}$, RAPHAEl Hirschi ${ }^{2,3}$, AND Ken'IChi Nomoto ${ }^{3,4}$ \\ ${ }^{1}$ Department of Physics and Astronomy, University of Victoria, BC V8W 3P6, Canada; swjones@uvic.ca \\ ${ }_{2}^{2}$ Astrophysics Group, Lennard-Jones Building, Keele University, Staffordshire ST5 5BG, UK \\ ${ }^{3}$ Kavli Institute for the Physics and Mathematics of the Universe (WPI), The University of Tokyo, Kashiwa, Chiba 277-8583, Japan \\ Received 2014 March 31; accepted 2014 October 10; published 2014 December 2
}

\begin{abstract}
In the ONeMg cores of 8.8-9.5 $M_{\odot}$ stars, neon and oxygen burning is ignited off-center. Whether or not the neon-oxygen flame propagates to the center is critical for determining whether these stars undergo Fe core collapse or electron-capture-induced $\mathrm{ONeMg}$ core collapse. We present more details of stars that ignite neon and oxygen burning off-center. The neon flame is established in a manner similar to the carbon flame of super-AGB stars, albeit with a narrower flame width. The criteria for establishing a flame can be met if the strict Schwarzschild criterion for convective instability is adopted. Mixing across the interface of the convective shell disrupts the conditions for the propagation of the burning front, and instead the shell burns as a series of inward-moving flashes. While this may not directly affect whether or not the burning will reach the center (as in super-AGB stars), the core is allowed to contract between each shell flash. Reduction of the electron fraction in the shell reduces the Chandrasekhar mass and the center reaches the threshold density for the URCA process to activate and steer the remaining evolution of the core. This highlights the importance of a more accurate treatment of mixing in the stellar interior for yet another important question in stellar astrophysics-determining the properties of stellar evolution and supernova progenitors at the boundary between electron capture supernova and iron core-collapse supernova.
\end{abstract}

Key words: nuclear reactions, nucleosynthesis, abundances - stars: AGB and post-AGB - stars: evolution stars: neutron - supernovae: general

Online-only material: color figures

\section{INTRODUCTION}

Differences in the pre-supernova structures of electron capture supernova (EC-SN) and iron core-collapse supernova (FeCCSN) progenitors, and hence the dynamics of the SN explosion itself, are postulated to be responsible for a number of observed phenomena. These phenomena include the orbital eccentricity of BeX systems (Knigge et al. 2011), the anticorrelations of silver and palladium abundances with other elements that have relatively well-known production sites (Hansen et al. 2012), Type IIn-P SNe (Anderson et al. 2012; Smith 2013), and some faint branch SNe with low ${ }^{56} \mathrm{Ni}$ ejecta. In spite of this motivation, the evolution of stars in the mass range $8-10 M_{\odot}$, wherein lies the transition between stars that will end their lives as EC-SNe and those that will produce FeCCSNe, is relatively under-represented in the literature. Also under-represented is the evolution of stars that ignite neon off-center (see Woosley et al. 1980; Nomoto 1980; Habets 1986; Nomoto \& Hashimoto 1988). The diversity of observations surrounding stars and $\mathrm{SNe}$, as well as improvements in the capabilities of numerical simulations, have led to renewed interest in these stars in recent years (Umeda et al. 2012; Tauris et al. 2013). These stars have compact cores bounded by a steep density gradient along which the SN shock is accelerated. The boundary between EC-SN and FeCCSN is an important ingredient for population synthesis and galactic chemical evolution; however, whether or not there are significant differences in the pre-SN structures of EC-SN and low-mass FeCCSN progenitors, and hence whether there are differences in their explosions and nucleosynthesis, is still to be investigated.

\footnotetext{
4 Hamamatsu Professor.
}

In our previous work (Jones et al. 2013), we presented new models of $8-12 M_{\odot}$ stars which suggest that there could be an evolutionary channel producing EC-SNe in addition to superAGB stars. These stars would be the most massive progenitors of EC-SNe and would ignite neon and oxygen burning offcenter where the maximum temperature has moved outward as neutrino emission produced a net cooling of the mildly electrondegenerate central regions. This off-center ignition of neon and oxygen is a characteristic also exhibited by the lowest-mass progenitors of FeCCSNe.

Timmes et al. (1994) suggested that should a star ignite neon sufficiently far from its center, its could become an EC-SN as opposed to an FeCCSN. The authors performed simulations of nuclear flames in degenerate oxygen-neon $(\mathrm{ONe})$ cores, providing tabulated flame speeds as a function of temperature and density; however, we note that the lower bound of density in the table is still an order of magnitude higher than the conditions under which neon is ignited in our simulations.

In super-AGB stars, it is carbon that is ignited off-center. It has been shown that in the limit of the strict Schwarzschild criterion for convection, a flame is established and the nuclear burning front propagates inward (toward the center of the star). Under these assumptions, the flame migrates inward from the ignition point because the peak energy generation from ${ }^{12} \mathrm{C}+{ }^{12} \mathrm{C}$ fusion resides below the peak temperature (Siess 2006; Denissenkov et al. 2013). This is due to the dependence of the fusion rate on the square of the ${ }^{12} \mathrm{C}$ abundance. Energy released by carbon burning heats the material, dragging the peak temperature inward, and thus the peak energy generation also moves inward.

Siess (2009) studied the effect of thermohaline mixing on the evolution and propagation of the carbon flame in super-AGB stars. Across the flame front, there is a steep mean molecular 
weight gradient transitioning between the unburnt composition $\left({ }^{12} \mathrm{C}\right.$ and $\left.{ }^{16} \mathrm{O}\right)$ and the composition after it has been processed by the flame $\left({ }^{20} \mathrm{Ne}\right.$ and $\left.{ }^{16} \mathrm{O}\right)$. The stratification of a fluid where material of higher mean molecular weight is situated atop material of lower mean molecular weight can induce mixing, depending on the steepness of the temperature gradient. Siess found that this thermohaline mixing at the carbon flame front, if sufficiently strong, could choke off the propagation of nuclear burning, and the carbon flame would thus fail to reach the center of the star.

Stellar evolution calculations treating thermohaline mixing as a diffusive process characterized by a salt-finger aspect ratio of $a \approx 7$ are able to reproduce the observed decrease of the surface ${ }^{12} \mathrm{C}$ abundance and ${ }^{12} \mathrm{C} /{ }^{13} \mathrm{C}$ ratio in RGB stars (Charbonnel \& Zahn 2007); however, recent two- and three-dimensional simulations of thermohaline mixing (Denissenkov 2010; Traxler et al. 2011) have shown the mixing to be much less efficient, characterized by a value of $a<1$. The simulations of Siess (2009), in which the carbon flame is quenched, had also assumed the same efficiency of thermohaline mixing, characterized by $a \approx 7$. Denissenkov et al. (2013), treating thermohaline mixing with the lower efficiency determined from multi-dimensional hydrodynamics simulations, find that this kind of mixing alone is not enough to quench the propagation of the carbon flame in super-AGB stars, and the flame successfully reaches the center of the star. Furthermore, Denissenkov and collaborators have tested the resilience of the carbon-burning flame when assuming a convective boundary mixing characterized by an exponentially decaying diffusion coefficient (Freytag et al. 1996; Herwig 2000 ) with values of $f_{\mathrm{CBM}}=0.014,0.007$, and 0.004 below the convective shell. In all cases, the flame was quenched even when accounting for additional heat transport in the boundary mixing region. The reason for the quenching of the flame in the presence of convective boundary mixing is the flattening of the ${ }^{12} \mathrm{C}$ abundance profile. The conditions for the propagation of the flame are no longer satisfied and the burning front does not reach the center. Under these assumptions, the super-AGB star can produce a hybrid white dwarf with an inner core of $\mathrm{CO}$ composition and an outer core of ONe composition. Chen et al. (2014) have shown that the quenching of the carbon flame in super-AGB stars leads to an increase in the theoretical upper limit to the initial mass of Type Ia SN progenitors when such hybrid white dwarfs are included. This could reduce the (already narrow) initial mass range for which EC-SNe are produced.

In this paper, we show the sensitivity of the neon-oxygen flame of low-mass massive stars to mixing across the formal Schwarzschild boundary at the base of the convection zone bounded by the flame. The mixing gives rise to a new evolutionary path to EC-SNe in which the core intermittently contracts between the recurrent quenching of neon-oxygen shell burning. The star is thus able to reach central densities dominated by the URCA-process, whose ability to remove electrons from the stellar plasma accelerates the evolution of the star toward an EC$\mathrm{SN}$, rather than an FeCCSN. For this reason, we refer to these stars which ignite neon and oxygen off-center and produce an EC-SN as failed massive stars (FMS).

\section{INPUT PHYSICS}

The models shown in this paper have been computed using the stellar evolution code MESA, using the same revision and input physics assumptions as in Jones et al. (2013), to which we refer the reader for more details. The only exception is the treatment of weak interaction rates, for which we use the new calculations of Toki et al. (2013) for the following three chains of weak interactions:

$$
\begin{aligned}
\text { (i) } & { }^{27} \mathrm{Al} \leftrightarrow{ }^{27} \mathrm{Mg} \leftrightarrow{ }^{27} \mathrm{Na} \\
\text { (ii }) & { }^{25} \mathrm{Mg} \leftrightarrow{ }^{25} \mathrm{Na} \leftrightarrow{ }^{25} \mathrm{Ne} \\
\text { (iii }) & { }^{23} \mathrm{Na} \leftrightarrow{ }^{23} \mathrm{Ne} \leftrightarrow{ }^{23} \mathrm{~F}
\end{aligned}
$$

which we showed in Jones et al. (2013) to make a non-negligible impact on the evolution of stars achieving densities greater than $\rho \approx 10^{9} \mathrm{~g} \mathrm{~cm}^{-3}$. Traversing one link in the chain requires the emission or absorption of an electron or positron, and always releases a neutrino with a mean free path much greater than the stellar radius. Calculating the electron fraction accurately is, of course, very important when simulating the evolution of stars that are supported by electron-degeneracy pressure. The strong neutrino cooling arising when beta-equilibrium is established (URCA process) between a parent and daughter nuclei from one such pair causes a steepening in the tail of the electron distribution function. As a result, the impact of electron captures on ${ }^{24} \mathrm{Mg}$ and ${ }^{20} \mathrm{Ne}$ that trigger the ignition of an oxygen deflagration and the collapse of the core will be felt at higher densities.

\section{IGNITION OF NEON AND OXYGEN BURNING AND DEVELOPMENT OF A CONVECTIVE ZONE}

We previously presented $8.8 M_{\odot}$ and $9.5 M_{\odot}$ stellar models in which neon and oxygen burning were ignited off-center in the degenerate core and proceeded to burn inward toward the stellar center (Jones et al. 2013). In the $8.8 M_{\odot}$ model, the burning failed to reach the center of the star and the calculation resulted in an EC-SN. Conversely, the burning successfully reached the center of the $9.5 M_{\odot}$ model, which would become an FeCCSN.

In our simulations of low-mass massive stars in which neon is ignited off-center, the situation is a little more complicated than that of the carbon flame in super-AGB stars. Rather than proceeding via the fusion of two similar nuclei, neon-burning is driven by photodisintegration. The key reactions during neon burning are

$$
\begin{aligned}
{ }^{20} \mathrm{Ne}+\gamma & \rightarrow{ }^{16} \mathrm{O}+\alpha \\
{ }^{16} \mathrm{O}+\alpha & \rightarrow{ }^{20} \mathrm{Ne}+\gamma \\
{ }^{20} \mathrm{Ne}+\alpha & \rightarrow{ }^{24} \mathrm{Mg}+\gamma .
\end{aligned}
$$

The neon photodisintegration reaction ${ }^{20} \mathrm{Ne}(\gamma, \alpha){ }^{16} \mathrm{O}$ has a $Q$ value of $-4.73 \mathrm{MeV}$ and is thus endothermic. When this reaction first becomes significant, the inverse reaction, ${ }^{16} \mathrm{O}(\alpha, \gamma){ }^{20} \mathrm{Ne}$, proceeds much faster, returning the energy to the stellar material and replenishing the ${ }^{20} \mathrm{Ne}$ abundance. When the temperature becomes high enough, however (see Figure 1), the $\alpha$ particle released is quickly captured by another ${ }^{20} \mathrm{Ne}$ nucleus, producing ${ }^{24} \mathrm{Mg}$. This reaction has a $Q$ value of $9.32 \mathrm{MeV}$ and is the primary energy source during neon burning.

At the point where the heat accumulates, ${ }^{20} \mathrm{Ne}$ is more efficient at capturing the $\alpha$ particles released via the photodisintegration of neon. The energy release is dictated by the photodisintegration rate and the burning proceeds effectively as the net reaction $2\left({ }^{20} \mathrm{Ne}\right) \rightarrow{ }^{16} \mathrm{O}+{ }^{24} \mathrm{Mg}+4.59 \mathrm{MeV}$. The dependence of the energy generation rate on the neon abundance is not as straightforward as in carbon burning, which is proportional to the square of the carbon abundance. Arnett (1974) and Woosley et al. (2002) have proposed that the energy generation rate during neon burning scales as $\epsilon \propto Y_{20}$ and $\epsilon \propto Y_{20}^{2} / Y_{16}$, 


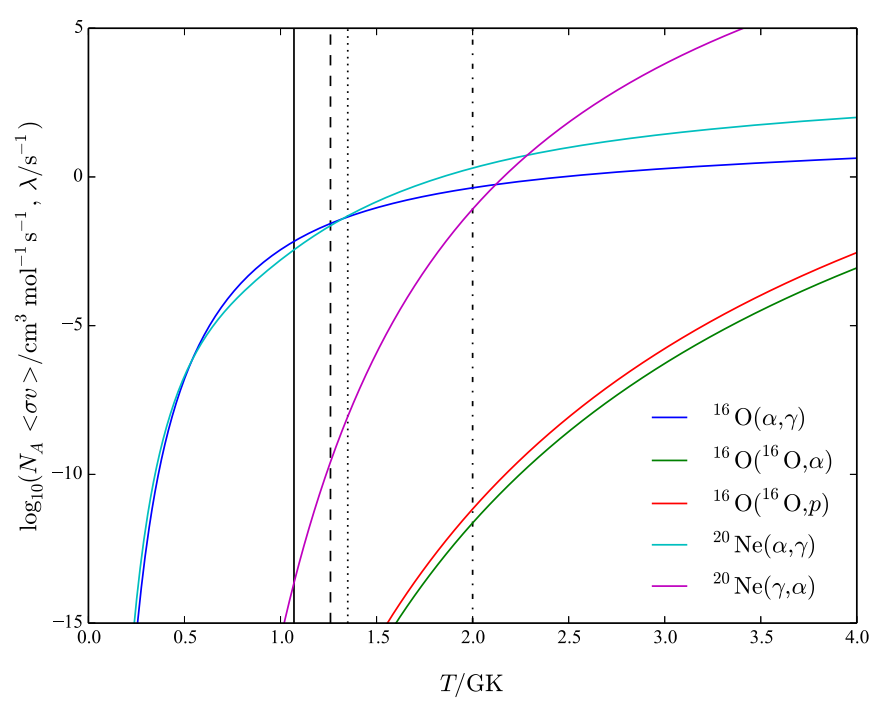

Figure 1. Reaction rates, $\lambda$ (one-body reactions), and $N_{A}\langle\sigma v\rangle$ (two-body reactions) of key neon and oxygen burning reactions as functions of temperature from the REACLIB compilation (Cyburt et al. 2010). The vertical black lines are drawn in order to facilitate comparison of the rates at temperatures of 1.07, $1.26,1.35$, and $2 \mathrm{GK}$ (see the text for details).

(A color version of this figure is available in the online journal.)

respectively. Both considerations omit the ${ }^{24} \mathrm{Mg}(\alpha, \gamma){ }^{28} \mathrm{Si}$ reaction, which we find to be important and which results in a scaling more like $\epsilon \propto Y_{20}^{1.4}$ (see the Appendix). In the Appendix, we employ a steady-state $\alpha$ particle abundance approximation (which we qualify) in order to look at the conditions under which the off-center neon-burning flame is established in our stellar models and how the energy generation rate is coupled to the stellar structure.

When the rate of energy generation from neon burning is high enough, the material above the region of nuclear burning becomes convectively unstable. When the fuel ignites off-center, as in the carbon flame of super-AGB stars (Siess 2006), the base of the convective zone does not develop at the coordinate of the peak temperature, but a small distance above it. This is because of the dependence of the luminosity on the temperature gradient, $L_{r} \propto d T / d r$. Of course, $d T / d r=0$ at the coordinate of the peak temperature and the criterion for convective instability,

$$
L_{r}>\frac{16 \pi a c G}{3} \frac{m T^{4}}{\kappa P} \nabla_{\mathrm{ad}},
$$

is satisfied at a point somewhere above the peak temperature where $\nabla_{\text {rad }}>\nabla_{\text {ad }}$ (Schwarzschild criterion).

In the convective region, a constant supply of fresh ${ }^{20} \mathrm{Ne}$ is being mixed down to the higher temperatures at the base. The temperature there is $1.26 \mathrm{GK}$, where the ${ }^{16} \mathrm{O}(\alpha, \gamma){ }^{20} \mathrm{Ne}$ and ${ }^{20} \mathrm{Ne}(\alpha, \gamma){ }^{24} \mathrm{Mg}$ reaction rates are incredibly similar (see blue, cyan, and vertical black dashed lines in Figure 1). As fresh ${ }^{20} \mathrm{Ne}$ is mixed down to this region, the constant release of energy raises the temperature enough to ignite oxygen burning, which proceeds by the following reactions:

$$
\begin{aligned}
& { }^{16} \mathrm{O}+{ }^{16} \mathrm{O} \rightarrow{ }^{31} \mathrm{P}+{ }^{1} \mathrm{H} \\
& { }^{31} \mathrm{P}+{ }^{1} \mathrm{H} \rightarrow{ }^{28} \mathrm{Si}+\alpha \\
& { }^{16} \mathrm{O}+{ }^{16} \mathrm{O} \rightarrow{ }^{28} \mathrm{Si}+\alpha .
\end{aligned}
$$

The development of a convective zone provides luminosity to support the outer layers of the core and temporarily halts the contraction of the core. The central regions thus expand and cool. The lifetime of the shell burning episode is lengthened while convection brings in fresh fuel to be burned at the base of the shell where the temperature is high.

\section{PROPAGATION OF THE BURNING FRONT TOWARD THE STELLAR CENTER}

A large range of the extent extra mixing at the convective boundary does not affect the qualitative evolutionary outcome of the $8.8 M_{\odot}$ model-an EC-SN (Jones et al. 2013). We now extend our analysis to include a case in which there is no convective boundary mixing across the base of the neon-burning shell (strict Schwarzschild criterion).

In the Introduction, we summarized the recent works of Siess (2009) and Denissenkov et al. (2013), which show that mixing across the flame front can destroy the conditions required for the persistence of a propagating nuclear flame in the stellar interior (the radial stratification of the peak energy production and temperature). Figure 2 shows the energy production due to the key neon- and oxygen-burning reactions during the peak of the first neon shell flash episode in the $8.8 M_{\odot}$ model. The top panel is the case assuming $f_{\mathrm{CBM}}=0.005$ below the shell flash convection zone and the bottom panel is for the case with $f_{\mathrm{CBM}}=0$ (no convective boundary mixing). Note the difference in scale of the $x$-axis for the plots.

In the case with $f_{\mathrm{CBM}}=0$ (pure Schwarzschild criterion, bottom panel), there are two distinct peaks in the energy production, separated by a thin region strongly depleted in neon. Just below this region (to the left in the plot), the temperature is about $1.35 \mathrm{GK}$ and the ${ }^{20} \mathrm{Ne}(\alpha, \gamma)^{24} \mathrm{Mg}$ and ${ }^{16} \mathrm{O}(\alpha, \gamma){ }^{20} \mathrm{Ne}$ reaction rates are still very similar (see blue, cyan, and vertical black dotted lines in Figure 1). The peak in energy production of each rate at this location traces the abundance of the fuel, and so the peak in ${ }^{20} \mathrm{Ne}(\alpha, \gamma){ }^{24} \mathrm{Mg}$ lies just below that of ${ }^{16} \mathrm{O}(\alpha, \gamma){ }^{20} \mathrm{Ne}$.

In the region where neon has been depleted, the temperature reaches $2 \mathrm{GK}$ and ${ }^{16} \mathrm{O}+{ }^{16} \mathrm{O}$ becomes significant. $\alpha$ particles are released by ${ }^{16} \mathrm{O}\left({ }^{16} \mathrm{O}, \alpha\right){ }^{28} \mathrm{Si}$ and ${ }^{16} \mathrm{O}\left({ }^{16} \mathrm{O}, p\right){ }^{31} \mathrm{P}(p, \alpha){ }^{28} \mathrm{Si}$. As the $\alpha$ particles are released in this way, ${ }^{16} \mathrm{O}(\alpha, \gamma){ }^{20} \mathrm{Ne}$ and ${ }^{20} \mathrm{Ne}(\alpha, \gamma){ }^{24} \mathrm{Mg}$ quickly turn ${ }^{16} \mathrm{O}$ into ${ }^{24} \mathrm{Mg}$ and because the ${ }^{20} \mathrm{Ne}(\alpha, \gamma){ }^{24} \mathrm{Mg}$ reaction is much quicker than ${ }^{16} \mathrm{O}(\alpha, \gamma){ }^{20} \mathrm{Ne}$ at this temperature (vertical black dot-dashed line in Figure 1), neon is completely depleted. ${ }^{24} \mathrm{Mg}(\alpha, \gamma){ }^{28} \mathrm{Si}$ proceeds at about half the rate of the ${ }^{20} \mathrm{Ne}(\alpha, \gamma){ }^{24} \mathrm{Mg}$ reaction and so the region starts to become enriched with ${ }^{24} \mathrm{Mg}$ and ${ }^{28} \mathrm{Si}$. It happens, then, that producing silicon from oxygen in this way is quicker than oxygen-oxygen fusion; however, it must rely upon the oxygenoxygen fusion reactions as the source of $\alpha$ particles.

After the neon is processed into ${ }^{24} \mathrm{Mg},{ }^{28} \mathrm{Si}$, and ${ }^{16} \mathrm{O}$ by the radiative pre-cursor neon flame, the burning moves inward toward the center because of its strong dependence on the neon abundance. Above the neon-depleted region (to the right in the plot), neon-burning energy production had previously boosted the luminosity above $L_{\text {crit }}$ and the material is convectively unstable, as we described earlier. The temperature in the convective region increases and oxygen-burning reactions $\left({ }^{16} \mathrm{O}+{ }^{16} \mathrm{O}\right)$ are activated.

In the case with $f_{\mathrm{CBM}}=0.005$, the evolution up to the development of the convective shell is the same for the case with $f_{\mathrm{CBM}}=0$, since there is no mixing. However, the situation evolves differently once the shell becomes convectively unstable. The extra mixing at the lower boundary of the convective shell homogenizes the composition across the thin 

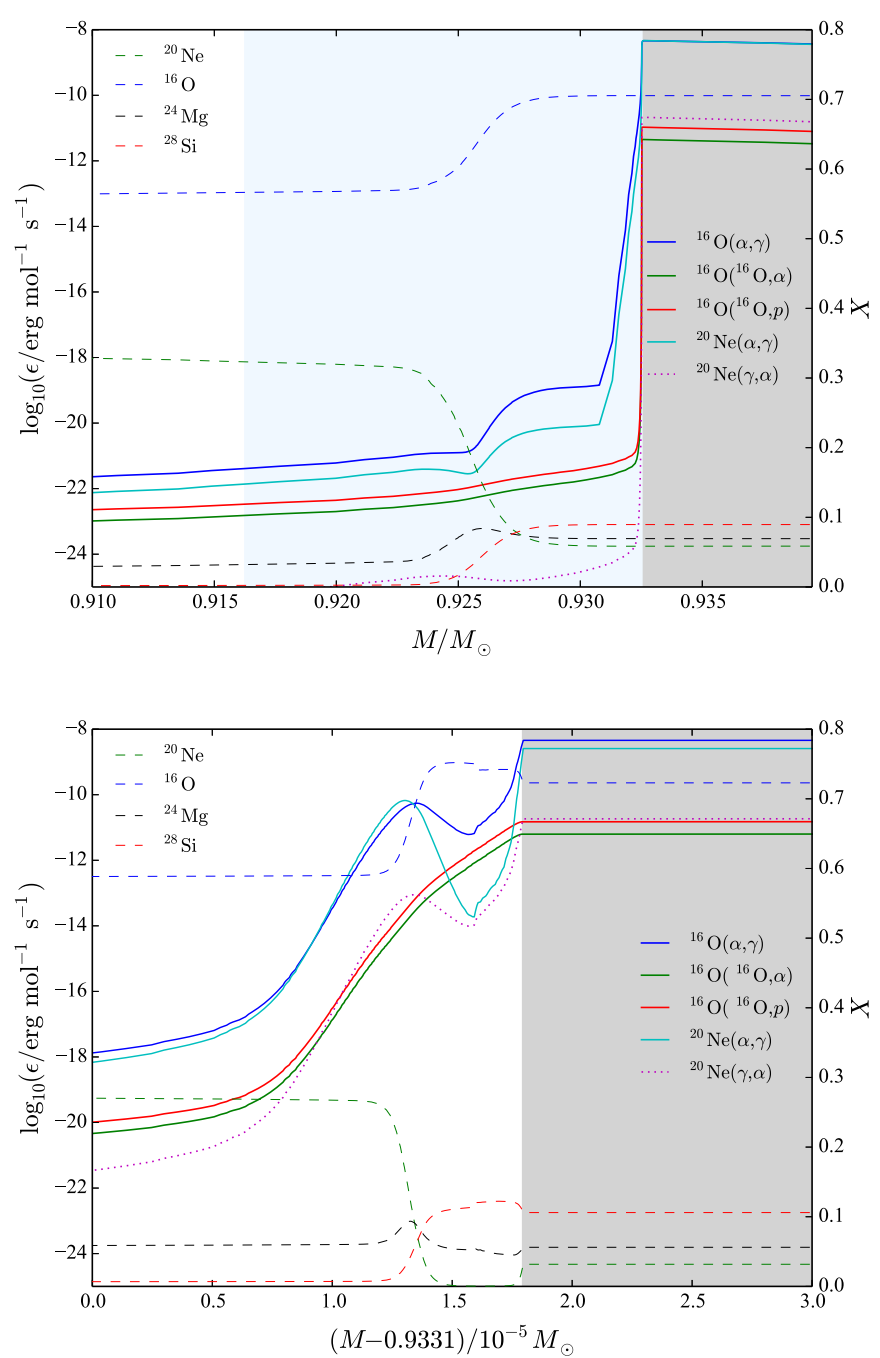

Figure 2. Energy production from key neon and oxygen burning reactions during the peak of the first neon shell flash in the $8.8 M_{\odot}$ model with $f_{\mathrm{CBM}}=0.005$ (top panel) and $f_{\mathrm{CBM}}=0$ (bottom panel) as functions of mass coordinate (absolute values are plotted, with negative quantities plotted with a dotted line style). The abundances of ${ }^{20} \mathrm{Ne}{ }^{16} \mathrm{O},{ }^{24} \mathrm{Mg}$, and ${ }^{28} \mathrm{Si}$ are plotted on the right axis. Note the difference in the scale of the $x$ axis for the two plots. Regions of convection are shaded gray and the extent of convective boundary mixing is shaded for the $f_{\mathrm{CBM}}=0.005$ model (top panel) in light blue.

(A color version of this figure is available in the online journal.)

radiative neon-burning shell with that in the convective oxygenburning shell. This mixing feeds the convective shell with neon, which proceeds to burn there via the net reaction $2\left({ }^{20} \mathrm{Ne}\right) \rightarrow{ }^{16}$ $\mathrm{O}+{ }^{24} \mathrm{Mg}+4.59 \mathrm{MeV}$ at much higher temperatures than are usually found during neon burning. This can be seen in the higher rate of energy production by ${ }^{20} \mathrm{Ne}(\alpha, \gamma){ }^{24} \mathrm{Mg}$-in particular, relative to that of ${ }^{16} \mathrm{O}(\alpha, \gamma){ }^{20} \mathrm{Ne}$-in the convective shell in the top panel of Figure $2\left(f_{\mathrm{CBM}}=0.005\right)$ compared to the bottom panel $\left(f_{\mathrm{CBM}}=0\right)$, and the higher abundances of ${ }^{20} \mathrm{Ne}$ and ${ }^{24} \mathrm{Mg}$.

In order for the flame to propagate, the energy production peak must be located below the temperature peak. Denissenkov et al. (2013) showed that in order for the peak energy production to reside below the peak temperature during the carbon-burning flame of super-AGB stars, the abundance gradient needs to be sufficiently steep to overcome the large temperature exponent $\left(\epsilon_{\text {nuc }} \propto \rho X^{2}\left({ }^{12} \mathrm{C}\right) T^{n}\right)$, where $n \approx 40$. In the absence of convective-boundary mixing, the neon-burning shell produces a steep gradient in the abundance of neon just below the ignition point. As we will demonstrate in the Appendix, the abundance gradient of neon is steep enough to establish a flame structure where the peak energy generation lies below the peak temperature. The flame is much thinner than in the case of offcenter carbon ignition in a $\mathrm{CO}$ core, and thus is more susceptible to disruption by convective boundary mixing.

As discussed above (and shown in greater detail in the Appendix), the peak in the energy generation of the radiative pre-cursor neon flame closely follows the sharp increase in the abundance of neon toward the center (see the left column of Figure 3 and compare to the right column of the same figure). If there is mixing at the convective boundary between the radiative layer and the convective shell, however, then the step in the $X\left({ }^{20} \mathrm{Ne}\right)$ profile is (1) smoothed out and (2) displaced toward the center of the star. With the mixing assumed to be characterized by an exponentially decaying diffusion coefficient with $f_{\mathrm{CBM}}=0.005$, the temperature at the new location of the step-up in neon abundance toward the center is $1.07 \mathrm{GK}$ $\left(\log _{10}(T / \mathrm{K}) \approx 9.03\right)$, and thus ${ }^{16} \mathrm{O}$ dominates ${ }^{20} \mathrm{Ne}$ in the capturing of any $\alpha$ particles (see Figure 1, vertical black solid line) and neon-burning barely proceeds at all.

The shell burning episode continues to bring neon and oxygen into the convective shell from the radiative layer below until both the convective region and the region in which the mixing had extended are rich in silicon-group composition $\left({ }^{34} \mathrm{~S},{ }^{30} \mathrm{Si}\right.$, and ${ }^{28} \mathrm{Si}$, in order of decreasing abundance by mass fraction) and depleted in ${ }^{16} \mathrm{O}$ and ${ }^{20} \mathrm{Ne}$. The convective shell persists until there is no longer sufficient luminosity to sustain it. Upon the extinction of the convective shell, the core contracts as described earlier. Neon burning re-ignites just below the extent of the boundary mixing where fuel is abundant as the core heats up. This behavior, where the shell exhausts its fuel and extinguishes, is the critical difference between the case with convective boundary mixing and the case without.

\subsection{Sensitivity of the Neon-burning Flame to $f_{C B M}$}

We have shown thus far that convective boundary mixing can disrupt the abundance gradients, and hence destroy the conditions under which a nuclear flame can smoothly propagate through the degenerate oxygen-neon core. By rearranging the formula for the diffusion coefficient in the convective boundary mixing region (Freytag et al. 1996; Herwig et al. 1997) to give an expression for $f_{\mathrm{CBM}}$ as

$$
f_{\mathrm{CBM}}=-\frac{2 z}{\lambda_{P, 0}\left(\ln D-\ln D_{0}\right)},
$$

we can estimate how small the $f_{\text {CBM }}$ parameter can be before the flame propagation may continue smoothly without intermittent quenching and contraction. In Equation (4), $\lambda_{P, 0}$ is the scale height of pressure at the lower boundary of the convective neonburning shell and $D_{0}$ is the diffusion coefficient at that same location. In order to obtain an order-of-magnitude estimate for the value of $f_{\mathrm{CBM}}$ that would disrupt the neon-burning flame, we take the radial distance from the convective boundary $z$ to be $10 \mathrm{~m}$ (approximately the width of the neon-burning flame front in our simulations; see Figure 3). We also need a value for the diffusion coefficient $D$ at this location. In our models, the mixing processes are frozen during neon-burning for diffusion coefficients less than about $D=10^{6} \mathrm{~cm}^{2} \mathrm{~s}^{-1}$, and thus we adopt this value. In fact, the value of $f_{\mathrm{CBM}}$ is not too sensitive to the exact value of $D$ and we find that the value of $f_{\mathrm{CBM}}$ 

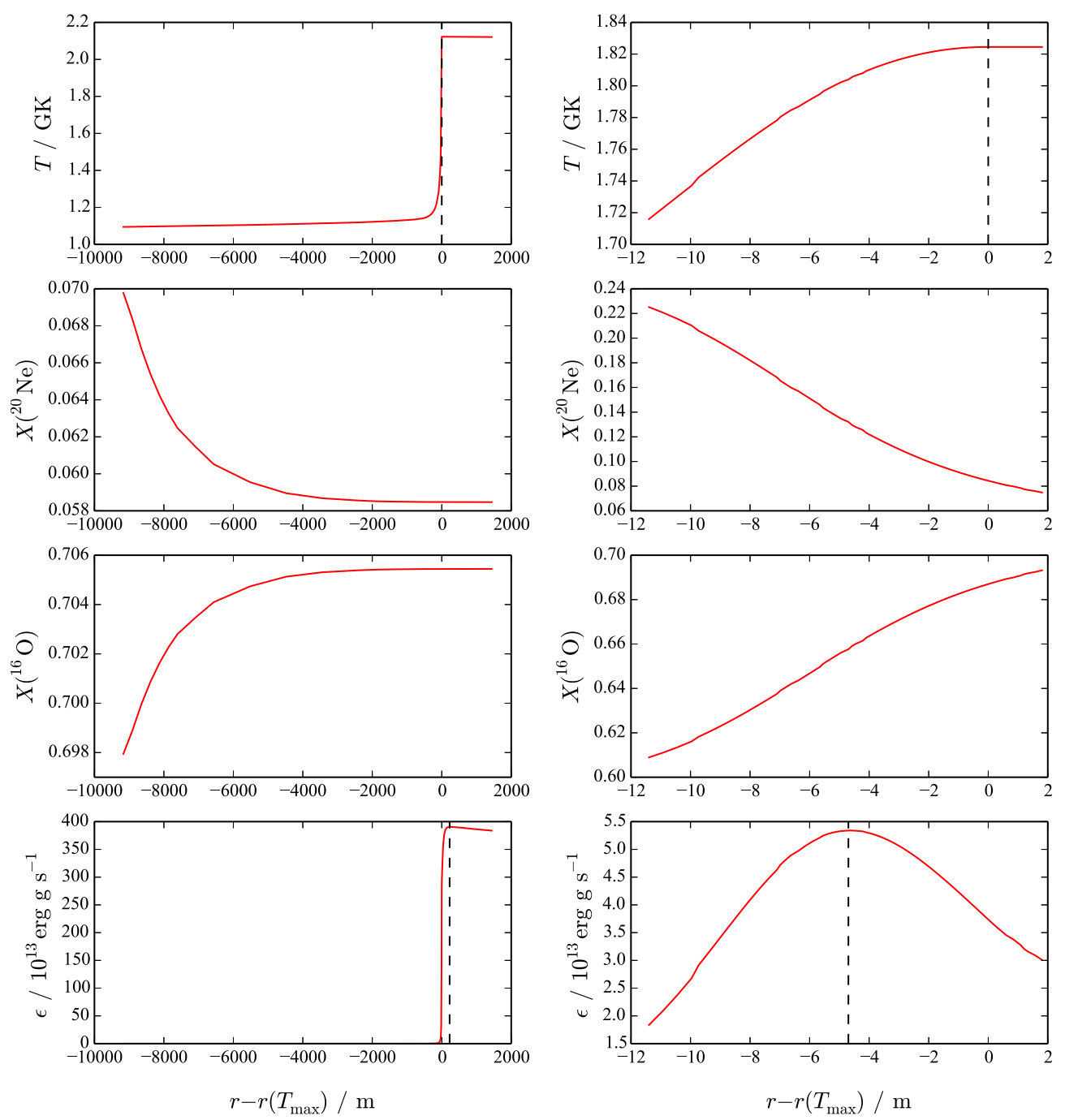

Figure 3. Properties of the model with $\left(f_{\mathrm{CBM}}=0.005\right.$; left column $)$ and without ( $f_{\mathrm{CBM}}=0$; right column $)$ convective boundary mixing $\left(f_{\mathrm{CBM}}=0\right)$ in the region of peak nuclear energy production $\epsilon$ due to neon burning. Dashed vertical lines show where quantities are a maximum. The peak energy generation due to neon burning (bottom panels) clearly sits above the peak temperature in the case with $f_{\mathrm{CBM}}=0.005$ (top left panel) and below the peak temperature in the case with $f_{\mathrm{CBM}}=0$ (top right panel). This difference is caused by the flattening of the ${ }^{20} \mathrm{Ne}$ and ${ }^{16} \mathrm{O}$ abundance profiles by the convective boundary mixing (left center panels; see right center panels).

(A color version of this figure is available in the online journal.)

required to interrupt the flame propagation is generally in the range $10^{-6} \lesssim f_{\mathrm{CBM}} \lesssim 10^{-5}$.

We have performed a similar calculation to the one above, this time for the carbon flame in a super-AGB stellar model, the width of which is of the order of $10 \mathrm{~km}$. The formula in Equation (4) shows that in order for convective boundary mixing (treated with the same diffusive model) to interfere with the carbon flame, the minimum value of the convective boundary mixing parameter would be $10^{-4} \lesssim f_{\mathrm{CBM}} \lesssim 10^{-3}$. This is consistent with Denissenkov et al. (2013), who showed that the carbon flame is quenched when $f_{\mathrm{CBM}}$ is as small as $7 \times 10^{-3}$.

\section{ELECTRON CAPTURE SUPERNOVA OR IRON CORE-COLLAPSE SUPERNOVA?}

We have shown that mixing across the neon-burning flame front in stars that ignite neon (and oxygen) off-center changes the manner in which neon burning migrates toward the stellar center. With no mixing (assuming only the Schwarzschild boundary), a flame is established and propagates (although we have not simulated the conductive propagation all the way) to the center of the star. When mixing across the convective boundary (more crucially, across the flame front) takes place, neon burning propagates in a discrete, step-wise manner with intermittent periods of contraction. Because of the finite mass of the fuel in the core, the number of shell flash episodes until the burning reaches the center is a function of the depth of the convective boundary mixing. This finding explains Figure 13 of Jones et al. (2013), where the simulations with larger values of $f_{\text {CBM }}$ experience fewer flashes (sharp deviations to the lower left of the figure) and stronger contraction following each flash.

As long as the convective boundary mixing (for which we do not yet know the strength) is strong enough to mix material across the flame front, a fraction of stars that ignite neon and oxygen burning off-center will reach the threshold density for the URCA process to be activated at their centers. These stars could hence produce EC-SNe as their contraction is accelerated toward the threshold density for electron captures by ${ }^{24} \mathrm{Mg}$ and 


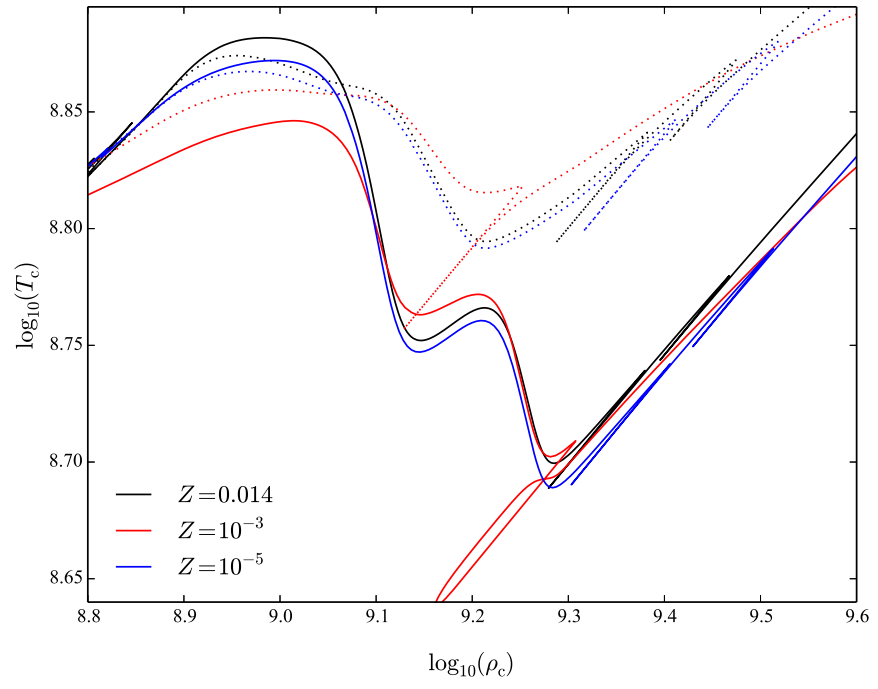

Figure 4. Central evolution of representative failed massive star models at three metallicities $\left(Z=0.014,0.001\right.$, and $\left.10^{-5}\right)$ governed by the URCA process during the neon shell flash phase. Dotted lines show the models where the weak rates of Oda et al. (1994) were used and solid lines are those where the new rates of Toki et al. (2013) including Coulomb corrections were used.

(A color version of this figure is available in the online journal.)

${ }^{20} \mathrm{Ne}$ to be activated. In addition, the cooling caused by the URCA process is stronger when using the new weak reaction rates calculated by Toki et al. (2013; see Figure 4). Each URCA pair produces a cooling front that moves outward from the center, leaving the temperature a factor of three cooler than the threshold temperature for neon ignition.

While the $9.5 M_{\odot}$ model in our set (see Jones et al. 2013) assumed exactly the same mixing assumptions as the $8.8 M_{\odot}$ model, the neon and oxygen burning had processed almost the entire core down to the center, and as a result the star would likely result in an FeCCSN. This is because the $9.5 M_{\odot}$ model ignites the off-center burning when its central density is much lower than in the $8.8 M_{\odot}$ model. As a result, the URCA process nuclei and the primary electron-capture fuel ${ }^{20} \mathrm{Ne}$ are destroyed before the threshold density for the URCA process is reached at the center. The slope of the evolution in the $\rho_{\mathrm{c}}-T_{\mathrm{c}}$ plane during the shell neon-burning phase (shown in Figure 4) is close to the adiabatic one, meaning that the same slope is found in all of the models. This means that if activation of the URCA process in these stars will indeed always produce an EC-SN, then there could be a relationship between the central density of the star at the time of off-center neon ignition and the fate of the star-EC-SN or FeCCSN.

\section{DISCUSSION AND CONCLUDING REMARKS}

In this paper, we have shown more details of stars that ignite neon and oxygen burning off-center in a degenerate core. If hydrodynamical instabilities at the base of the convection zone trailing the narrow flame front induce mixing across the front, then the nuclear burning migrates toward the center in a stepwise manner with intermittent periods of contraction. If there is strictly no mixing across the front, then the propagation ensues as a flame chasing the abundance gradient of ${ }^{20} \mathrm{Ne}$. For a convective boundary mixing model based on an exponentially decaying diffusion coefficient (e.g., Freytag et al. 1996; Herwig et al. 1997), quenching of the flame can be achieved for diffusion coefficient e-folding lengths of around $10^{-6} \lambda_{P}$ or larger. The equivalent e-folding length for quenching of the carbon flame in
super-AGB stars is greater by about three orders of magnitude. This e-folding length of the diffusion coefficient required to quench the neon flame could be considered small in comparison, and thus (if the neon flame is subjected to the same types of hydrodynamical instabilities as the carbon flame) if the carbon flame can be quenched, it is very likely that the neon flame will experience quenching. Of course, these results are limited by the assumption that the convective boundary mixing model that we have adopted is representative of the hydrodynamic processes that induce the mixing at such a boundary in stars. Threedimensional hydrodynamics simulations of the neon flame, capturing the turbulent motion such as the ones presented in Meakin \& Arnett (2007), Herwig et al. (2011, 2014), and Viallet et al. (2013), are needed to better constrain the width of the flame and, more importantly, the type of mixing that occurs across the boundary.

We have shown that it is the intermittent periods of contraction due to the quenching of the burning front by convective boundary mixing that cause an $8.8 M_{\odot}$ model to reach the threshold density for the URCA process to be activated. The model contracts to higher densities following each shell flash due to the reduction in the electron fraction in the oxygenburning shell. The ultimate removal of electrons by the URCA process drives the evolution toward an EC-SN.

In order to progress further with this study and make predictions about how FMS may contribute to the EC-SN rate, we require constraints on the behavior of both the flame itself and the strength of the mixing across the flame front. Multi-dimensional hydrodynamics simulations should provide us with the insight required to make a firm conclusion. If the mixing is indeed strong enough to cause the step-wise propagation of the flame, the results of Tauris et al. (2013) suggest that EC-SNe from stars in binary configurations could even produce type Ic explosions.

We thank the referee for comments that have significantly improved our manuscript. The research leading to these results has received funding from the European Research Council under the European Union's Seventh Framework Programme (FP/ 2007-2013)/ERC grant agreement No. 306901. R.H. thanks the Eurocore project Eurogenesis for support. K.N., R.H., and S.J. acknowledge support from the World Premier International Research Center Initiative (WPI Initiative), MEXT, Japan.

\section{APPENDIX \\ DISCUSSION AND DERIVATIONS OF NEON-BURNING EQUATIONS}

The rate of change of the helium abundance during neon burning is given by the expression

$$
\begin{aligned}
\dot{Y}_{\alpha}= & -Y_{\alpha}\left(Y_{20} \lambda_{20 \alpha \gamma}+Y_{16} \lambda_{16 \alpha \gamma}+Y_{24} \lambda_{24 \alpha \gamma}\right) \\
& +Y_{20} \lambda_{20 \gamma \alpha}+Y_{24} \lambda_{24 \gamma \alpha} .
\end{aligned}
$$

If the alpha abundance is in a steady state, then $\dot{Y}_{\alpha} \approx 0$ and Equation (A1) gives an expression for the $\alpha$ particle abundance as

$$
Y_{\alpha}=\frac{Y_{20} \lambda_{20 \gamma \alpha}+Y_{24} \lambda_{24 \gamma \alpha}}{Y_{20} \lambda_{20 \alpha \gamma}+Y_{16} \lambda_{16 \alpha \gamma}+Y_{24} \lambda_{24 \alpha \gamma}} .
$$

The quantities $\lambda$ are the reaction rates with units of either $\mathrm{g} \mathrm{s}^{-1}$ $\left(\rho N_{\mathrm{A}}\langle\sigma v\rangle\right)$ for the two-body reactions or just $\mathrm{s}^{-1}$ for the photodisintegration reactions. At neon-burning temperatures of about 


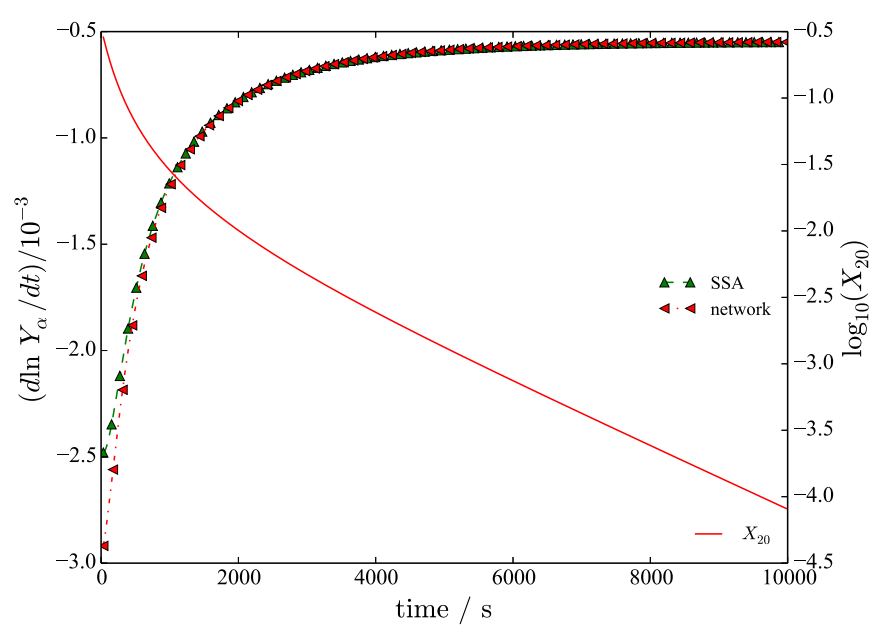

Figure 5. Time-derivatives of the $\alpha$ particle abundance as given directly by a one-zone neon burning network calculation (red sideways triangle) and by assuming a steady-state abundance of $\alpha$ particles for the same calculation (green upright triangle). The formula for $d \ln Y_{\alpha} / d t$ under the steady-state $\alpha$ particle abundance approximation is given in Equation (A3). $X_{20}$ is the mass fraction of ${ }^{20} \mathrm{Ne}$ and is drawn with a solid red line. The two time derivatives agree well, showing that a steady-state $\alpha$ particle abundance is a good approximation when analyzing the behavior of neon burning.

(A color version of this figure is available in the online journal.)

$1.8 \mathrm{GK}, \lambda_{20 \gamma \alpha} / \lambda_{24 \gamma \alpha} \approx 10^{10}$, and thus the ${ }^{24} \mathrm{Mg}(\gamma, \alpha)^{20} \mathrm{Ne}$ reaction can be considered negligible. Taking the logarithm and then the time derivative of both sides of Equation (A2) yields the relation

$$
\frac{d \ln Y_{\alpha}}{d t}=\frac{d \ln Y_{20}}{d t}-\frac{d}{d t} \ln \left(Y_{20} \lambda_{20 \alpha \gamma}+Y_{16} \lambda_{16 \alpha \gamma}+Y_{24} \lambda_{24 \alpha \gamma}\right) .
$$

In Figure 5, the quantity $Y \alpha / d t$ has been calculated two different ways and is shown as a function of time. The line labelled "netwok" has been determined numerically in a onezone nucleosynthesis simulation with $T_{9}=1.82 \mathrm{GK}$ and $\rho=2.79 \times 10^{7} \mathrm{~g} \mathrm{~cm}^{-3}$; the line labelled "SSA" was calculated using the formula in Equation (A3). These are the conditions under which the neon shell burns in our $8.8 M_{\odot}$ stellar model. Figure 5 shows that the steady-state $\alpha$ particle abundance approximation is indeed appropriate for the analysis of neon burning behavior.

It is important to note at this point that a steady-state abundance of $\alpha$ particles is not assumed within the reaction network or any other part of the MESA code, which performs a network integration that is fully coupled to the structure and mixing operators. We use the steady-state $\alpha$ particle abundance only as a means to describe the behavior of neon burning - and in particular its interaction with convective boundary mixing-in the stellar models that we have calculated.

Following the derivation of Arnett (1974), under the assumption of a steady-state $\alpha$ particle abundance, the time derivatives of the other abundances are given by

$$
\begin{aligned}
& \dot{Y}_{16}=Y_{20} \lambda_{20 \gamma \alpha}(1-A), \\
& \dot{Y}_{20}=Y_{20} \lambda_{20 \gamma \alpha}(A-1-B), \\
& \dot{Y}_{24}=Y_{20} \lambda_{20 \gamma \alpha}(B-C), \\
& \dot{Y}_{28}=Y_{20} \lambda_{20 \gamma \alpha} C,
\end{aligned}
$$

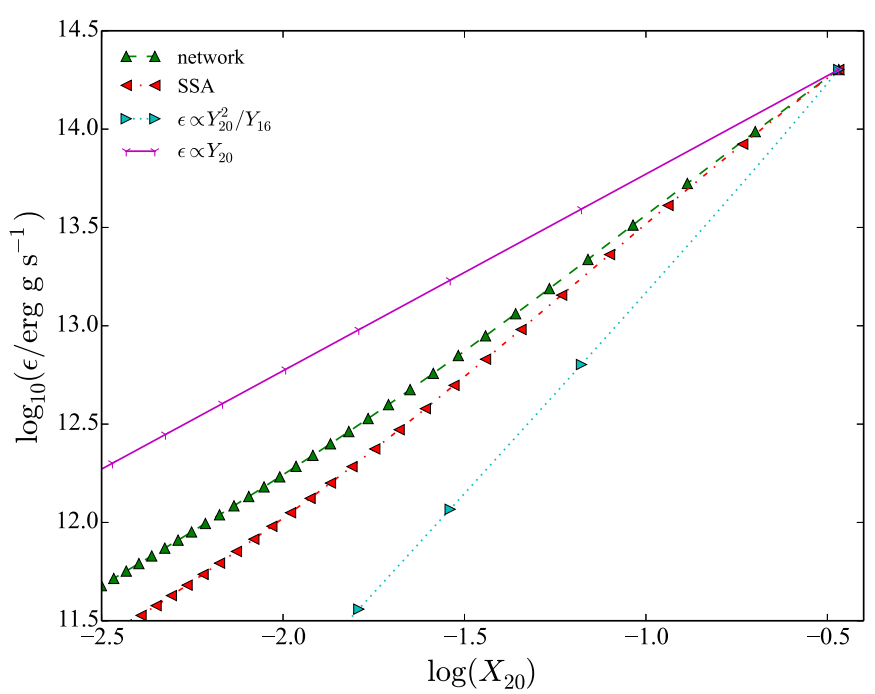

Figure 6. Energy generation rate during neon burning as a function of the ${ }^{20} \mathrm{Ne}$ mass fraction. The numerically evaluated rate from the network calculation is shown in green (upright triangles) along with the energy generation predicted by the analytical formula in Equation (A7) assuming a steady-state abundance of $\alpha$ particles (red sideways triangles). For comparison, we show the energy generation rates given by the relations in Woosley et al. (2002) and Arnett (1974) which scale as $\epsilon \propto Y_{20}^{2} / Y_{16}$ and $\epsilon \propto Y_{20}$, respectively.

(A color version of this figure is available in the online journal.)

where $A, B$, and $C$ are defined as

$$
\begin{aligned}
& A=\frac{Y_{16} \lambda_{16 \alpha \gamma}}{\xi} \\
& B=\frac{Y_{20} \lambda_{20 \alpha \gamma}}{\xi} \\
& C=\frac{Y_{24} \lambda_{24 \alpha \gamma}}{\xi},
\end{aligned}
$$

and $\xi$ is the denominator in the right-hand side of Equation (A2),

$$
\xi=Y_{20} \lambda_{20 \alpha \gamma}+Y_{16} \lambda_{16 \alpha \gamma}+Y_{24} \lambda_{24 \alpha \gamma} \text {. }
$$

An expression for the energy generation during neon burning $\left(\epsilon \propto \sum_{i} \dot{Y}_{i} B_{i}\right.$, where $B_{i}$ is the binding energy of a nucleus of type $i$ ) can now be formulated using the time-derivatives of the key abundances (Equation (A4)) and the fact that $A+B+C=1$ (which we have also confirmed numerically). The resulting expression is

$$
\ln \epsilon=\ln Y_{20}+\ln \lambda_{20 \gamma \alpha}+\ln \phi-\ln \zeta+D
$$

where

$$
\phi=N Y_{24}\langle\sigma v\rangle_{24 \alpha \gamma}+[N+M] Y_{20}\langle\sigma v\rangle_{20 \alpha \gamma},
$$

$D$ is a constant, and $\zeta=\xi / \rho$. In Figure 6 , we show the energy generation rate given by Equation (A7) compared to the rate evaluated numerically by the reaction network. We also show in Figure 6 the scaling of the neon burning energy generation with the ${ }^{20} \mathrm{Ne}$ abundance as formulated by Woosley et al. (2002) and Arnett (1974), which scale as $\epsilon \propto Y_{20}^{2} / Y_{16}$ and $\epsilon \propto Y_{20}$, respectively, and fail to reproduce the trend in our network calculation. The peak energy generation for each approximation has been normalized to the peak energy generation from the reaction network in order to more clearly highlight the differences between the different cases. Although 
we follow an almost identical derivation to Arnett (1974), the main difference is that Arnett assumes that the ${ }^{24} \mathrm{Mg}(\alpha, \gamma){ }^{28} \mathrm{Si}$ reaction is negligible (i.e., $C=0$ in Equation (A5)), which is not the case in our calculations. From the network calculation, the slope down to about $X_{20}=0.01$ is close to $\epsilon \propto Y_{20}^{1.4}$.

In order for the conditions for a flame structure to be established, it must be possible that

$$
\frac{\partial \ln \epsilon}{\partial \ln T}=\frac{\partial \ln Y_{20}}{\partial \ln T}+\frac{\partial \ln \lambda_{20 \gamma \alpha}}{\partial \ln T}+\frac{\partial \ln \phi}{\partial \ln T}-\frac{\partial \ln \zeta}{\partial \ln T}<0 .
$$

We have evaluated each derivative in Equation (A9) numerically from our stellar model and although none of the terms are negligible, it is the first two terms (involving $Y_{20}$ and $\lambda_{20 \gamma \alpha}$ ) that dominate. $\partial \ln \lambda_{20 \gamma \alpha} / \partial \ln T$ remains reasonably fixed around the flame location at about 42. The abundance gradient of ${ }^{20} \mathrm{Ne}$ resulting from the thin flame front is steep enough to dominate over the gradient of the ${ }^{20} \mathrm{Ne}(\gamma, \alpha){ }^{16} \mathrm{O}$ rate and together with the other terms results in $\partial \ln \epsilon / \partial \ln T<0$ across a region of a few meters. The flame width is much thinner for off-center neon burning compared to off-center carbon burning, making it more susceptible to destruction by convective boundary mixing (see Section 4.1).

\section{REFERENCES}

Anderson, J. P., Habergham, S. M., James, P. A., \& Hamuy, M. 2012, MNRAS, 424, 1372

Arnett, W. D. 1972, ApJ, 176, 699

Arnett, W. D. 1974, ApJ, 193, 169

Charbonnel, C., \& Zahn, J.-P. 2007, A\&A, 467, L15
Chen, M. C., Herwig, F., Denissenkov, P. A., \& Paxton, B. 2014, MNRAS, 440, 1274

Cyburt, R. H., Amthor, A. M., Ferguson, R., et al. 2010, ApJS, 189, 240

Denissenkov, P. A. 2010, ApJ, 723, 563

Denissenkov, P. A., Herwig, F., Truran, J. W., \& Paxton, B. 2013, ApJ, 772,37

Freytag, B., Ludwig, H.-G., \& Steffen, M. 1996, A\&A, 313, 497

Habets, G. M. H. J. 1986, A\&A, 167, 61

Hansen, C. J., Primas, F., Hartman, H., et al. 2012, A\&A, 545, A31

Herwig, F. 2000, A\&A, 360, 952

Herwig, F., Bloecker, T., Schoenberner, D., \& El Eid, M. 1997, A\&A, 324, L81

Herwig, F., Pignatari, M., Woodward, P. R., et al. 2011, ApJ, 727, 89

Herwig, F., Woodward, P. R., Lin, P.-H., Knox, M., \& Fryer, C. 2014, ApJL, 792, L3

Jones, S., Hirschi, R., Nomoto, K., et al. 2013, ApJ, 772, 150

Knigge, C., Coe, M. J., \& Podsiadlowski, P. 2011, Natur, 479, 372

Meakin, C. A., \& Arnett, D. 2007, ApJ, 667, 448

Nomoto, K. 1980, in Texas Workshop on Type I Supernovae, ed. J. C. Wheeler (Austin, TX: Univ. Texas), 164

Nomoto, K., \& Hashimoto, M. 1988, PhR, 163, 13

Oda, T., Hino, M., Muto, K., Takahara, M., \& Sato, K. 1994, ADNDT, 56,231

Siess, L. 2006, A\&A, 448, 717

Siess, L. 2009, A\&A, 497, 463

Smith, N. 2013, MNRAS, 434, 102

Tauris, T. M., Langer, N., Moriya, T. J., et al. 2013, ApJL, 778, L23

Timmes, F. X., Woosley, S. E., \& Taam, R. E. 1994, ApJ, 420, 348

Toki, H., Suzuki, T., Nomoto, K., Jones, S., \& Hirschi, R. 2013, PhRvC, 88, 015806

Traxler, A., Garaud, P., \& Stellmach, S. 2011, ApJL, 728, L29

Umeda, H., Yoshida, T., \& Takahashi, K. 2012, PTEP, 2012, 010000

Viallet, M., Meakin, C., Arnett, D., \& Mocák, M. 2013, ApJ, 769, 1

Woosley, S. E., Heger, A., \& Weaver, T. A. 2002, RvMP, 74, 1015

Woosley, S. E., Weaver, T. A., \& Taam, R. E. 1980, in Texas Workshop on Type I Supernovae, ed. J. C. Wheeler (Austin, TX: Univ. Texas), 96 